\title{
Brain composition and scaling in social bee species differing in body size
}

\author{
Vishwas GowdA $^{1,2}$, Wulfila GronenBERG ${ }^{1}$ \\ ${ }^{1}$ Department of Neuroscience, The University of Arizona, 1040 E. 4th Street, Tucson, AZ 85721, USA \\ ${ }^{2}$ Graduate Interdisciplinary Program Entomology and Insect Science, The University of Arizona, Tucson, AZ 85721 , \\ USA
}

Received 22 August 2018 - Revised 16 July 2019 - Accepted 1 September 2019

\begin{abstract}
We compare four honeybee and one bumblebee species with similar behaviors and ecological requirements but large differences in body size. The bees show allometric brain-body size relationships and scaling exponents similar to those found in vertebrates. Compared with three Asian honeybee species, the European honeybee Apis mellifera feature larger brains than expected for their body mass. Overall, the brains show moderate regional variation across species with two pronounced differences: A. dorsata have an enlarged visual lamina, possibly an adaptation for crepuscular vision. Second, the mushroom bodies are larger and comprise more intrinsic neurons in bumblebees than in honeybees. While these findings might suggest more advanced sensory associations in bumblebees, it is currently not possible to test this notion in the absence of quantitative and comprehensive behavioral comparisons across bee species.
\end{abstract}

allometric scaling / morphometry / mushroom body / honeybees / bumblebees

\section{Abbreviations \\ MB Mushroom body}

\section{INTRODUCTION}

In vertebrates, an allometric relationship exists between the brain and body mass (Jerison, 1973; Striedter, 2005). The allometric exponent of this logarithmic relationship varies across vertebrate taxa, and exact values are a matter of debate (Martin 1981). Likewise, absolute brain size increases in insects, while relative brain size decreases with increasing body size (Neder 1959; Wehner et al. 2007). Larger brains or brain

Electronic supplementary material The online version of this article (https://doi.org/10.1007/s13592-019-00685-w) contains supplementary material, which is available to authorized users.

Corresponding author: V. Gowda, vishwas@email.arizona.edu

Manuscript editor : Bernd Grünewald components generally comprise more and/or larger neurons (Herculano-Houzel et al. 2014), and some studies suggest that they may provide animals with enhanced cognitive ability and enlarged behavioral repertoires (Lefebvre et al. 2004). However, behavioral repertoires and complexity are notoriously difficult to quantify, and the presence of a larger brain may not necessarily translate into greater cognitive abilities. Environmental factors, diet, or social and other behaviors all can have independent implications on the brain and brain component size (Dunbar and Shultz 2007; Byrne and Bates 2007; Healy and Rowe 2007). It would therefore be advantageous to address the question of body size-related brain modifications within animals that differ little in traits other than body size.

Here, we ask the question if brain-body scaling relationships established in vertebrates are similar 
in insects. Specifically, we chose four closely related honeybee and one bumblebee species that differ in body size but are behaviorally similar and have similar ecological requirements (Figure 1a). While the four honeybee species show relatively restricted, species-specific body size ranges, bumblebees (B. impatiens) cover a much larger range of individual body sizes (Goulson et al. 2002), overlapping with the three larger honeybee species, but including individuals much larger than any honeybee (Figure 1). The four honeybee species show similar social behaviors [division of labor, communication of food location and quality, dance language, etc. (Oldroyd and Wongsiri 2006)]. The similarities among these five bee species should allow establishing relevant brainbody size correlations little encumbered by behavioral and ecological differences. We volumetrically compare the bees' brains and brain components and the number of neurons in two well-delineated cell body regions in the five species. We focus on sensory brain compartments, which generally scale in size and complexity with the respective insect's sensory capacities (Chittka and Niven 2009; Stöckl et al. 2016b), and on the mushroom body (MB), which supports advanced cognitive functions (reviewed in Strausfeld 2012; Fahrbach 2006). We also compare the size of the prothoracic ganglion in the four honeybee species, assuming that the ventral nerve cord performs more basic functions and is much less involved in individual cognitive capabilities, compared with the brain, and thus possibly represents more closely the actual relationship between the nervous system and body size.

\section{MATERIALS AND METHODS}

Here, we present the Methods in a condensed form. A more detailed Methods section can be found in the Electronic Supplementary Material.

\subsection{Specimens/sample collection}

Workers of three Asian honeybee species were collected on and around the campus of the Indian Institute of Science, Bangalore, India (Figure 1a): the Indian honeybee (A. cerana), the dwarf honeybee (A. florea), and the giant honeybee
(A. dorsata). European honeybees (Apis mellifera carnica) were raised and collected at the Carl Hayden Bee Laboratory, USDA ARS, Tucson, Arizona. All bees were of unknown age and were foraging for either nectar or water, but not for pollen. Bumblebee colonies (Bombus impatiens) were purchased from Biobest Inc. (McFarland, CA, USA).

\subsection{Histology and morphometry}

The bees' head widths were measured using calipers, and the animals' combined head and thorax were weighed. This measure (combined head and thorax mass) is independent of the degree of the bees' satiation, and body mass shows a stronger correlation with brain volume than head width [Mares et al. (2005); Riveros and Gronenberg (2010)]. To make our head-thorax mass data comparable to previously published studies, we measured and compared total body mass and head-thorax mass in a separate set of $21 \mathrm{~A}$. mellifera and $78 \mathrm{~B}$. impatiens bees. This data set is not included in the brain data set but has been added as Electronic Supplementary Material. These data were used to calculate correction factors $(1.79 \pm 0.35$ for A. mellifera and $1.85 \pm 0.25$ for $B$. impatiens ) for converting our head-thorax mass data to total body mass data commonly published in other studies.

Brains were dissected and fixed (4\% formaldehyde in $0.1 \mathrm{M}$ cacodylate buffer; $\mathrm{pH}$ 6.8) overnight, rinsed in buffer, and then stained in $1 \%$ aqueous osmium tetroxide solution for $2 \mathrm{~h}$ at $4{ }^{\circ} \mathrm{C}$. Prothoracic ganglia of the four honeybee species (but not of bumblebees) were removed from the bees' thoraces and processed like the brains. Brains and ganglia were then rinsed in water, dehydrated, embedded in Spurr's low viscosity embedding medium (Electron Microscopy Science; Hartfield, PA), polymerized at $70{ }^{\circ} \mathrm{C}$, sectioned on a sliding microtome at $15 \mu \mathrm{m}$ thickness, and coverslipped using Cytoseal (Thermo Scientific).

Brains and brain components were traced and measured using the Photoshop CS5 (Adobe) pixel counting tool. Brains were sampled at $30 \mu \mathrm{m}$ (A. florea, A. cerana, and A. mellifera) or $45 \mu \mathrm{m}$ intervals (A. dorsata and B. impatiens) 

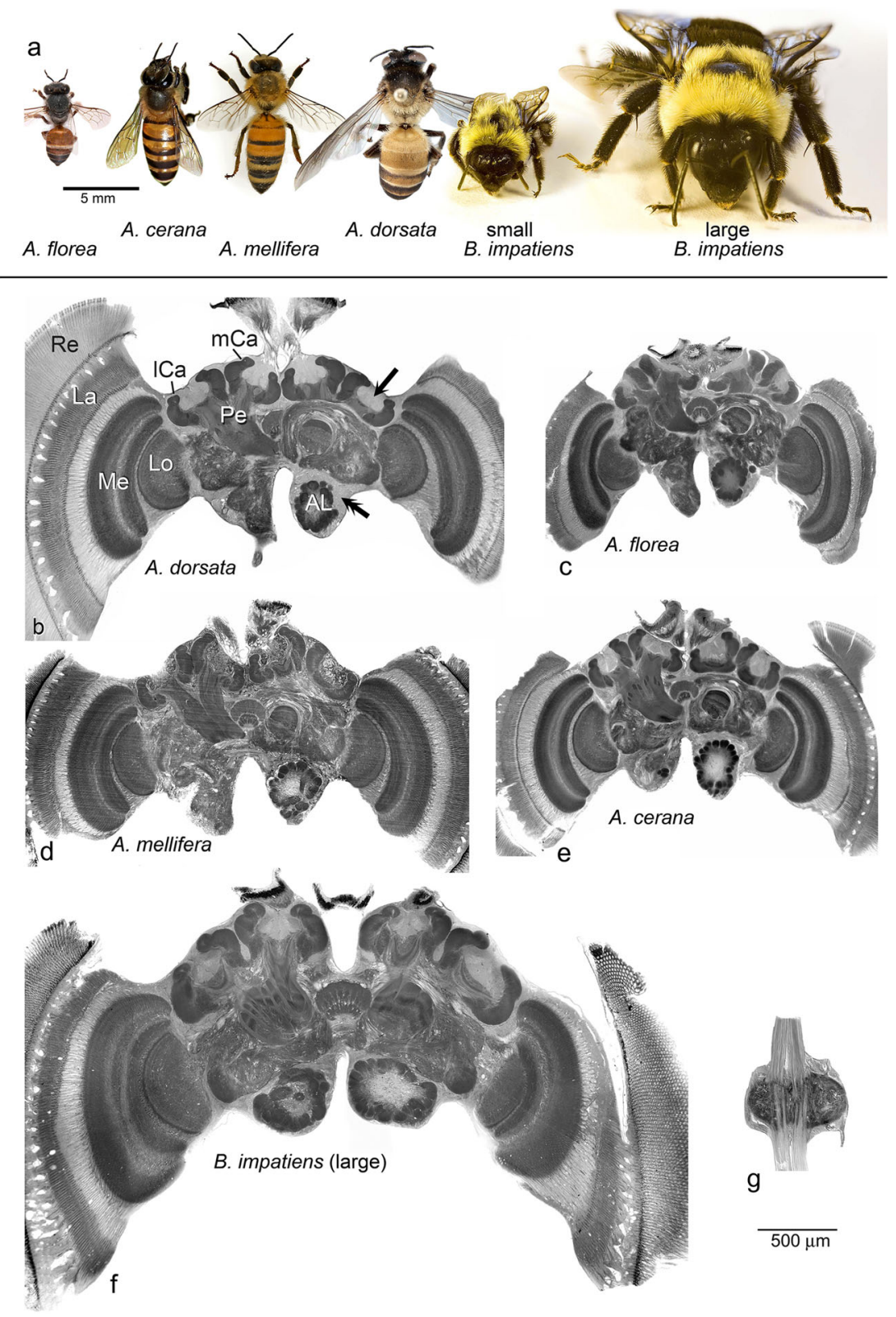

Figure 1. Photographs of the five bee species (a) and photomicrographs of their respective brains (b-f; photomontages representing major brain components). $g$ Prothoracic ganglion of $A$. dorsata. Brain components labeled in (b): antennal lobe (AL), lateral calyx (lCa), medial calyx (mCa), MB peduncle (Pe), lamina (La), lobula (Lo), medulla (Me), retina (Re). Photo credits (a): A. florea: Simon Hinkley \& Ken Walker(Museum Victoria; http://www.padil.gov.au/pests-and-diseases/pest/main/135537/2446); A. cerana: Charles Lam (http://www. inaturalist.org/taxa/338194-Apis-cerana); A. mellifera: Gary R. McClellan (http://bugguide.net/node/view/603265 ); Apis dorsata: Ken Walker (Museum Victoria; http://www.padil.gov.au/pests-and-diseases/pest/main/135533/65).

based on the "Cavalieri" method (Gundersen and Jensen 1987). Brain volumes were calculated from the area measurements multiplied by the section thickness. The volumes of each brain and 
its components (antennal lobes, medulla, lobula, lamina, MB calyx and lobes, and central body; but excluding the retina; indicated in Figure 1b) were thus measured in addition to the sum of all other neuropils and cell bodies (here referred to as "rest brain"). Relative volumes of brain components were calculated by dividing the respective volume by the overall volume of the brain including the cell bodies.

\subsection{Estimating cell number and cell body volume}

We compared the neuron cell body volumes in two regions of the brain where cell body clusters could be distinctly defined: the antennal lobes (double-headed arrow in Figure 1b) and the MB Kenyon cells within the lateral calyx (arrow in Figure 1b). The calyx cups comprise two classes of Kenyon cell somata that we here refer to as small $\left(\mathrm{KC}_{\mathrm{sm}}\right)$ and large Kenyon cells $\left(\mathrm{KC}_{\mathrm{lg}}\right)$, respectively. We measured the volume of both soma clusters and estimated the number and volume of the two kinds of Kenyon cells separately [Figures 2 and 3; Table I]. To estimate the neuron numbers, we counted the numbers of nuclei in a probe area $\left(20 \times 20 \mu^{2}\right)$ sampling every $9 \mu \mathrm{m}$ from serial sections. From these nucleus counts and the respective sampled volume, the total cell number for the given region was estimated. The mean volume of the cell bodies was extrapolated from the neuron number and sampled volume, assuming sphere-shaped cell bodies and a dense packaging taking up $90 \%$ of the respective volume (see Electronic Supplemental Material for more detail). We want to point out that our estimates of cell body volumes are not independent measures but are derived from the cell count and the volume of the respective cell body regions.

\subsection{Data analysis}

Correlations between brain volumes and head/ thorax mass or head width were log-transformed and compared using linear regressions and ANCOVA. The mean brain and brain component volumes were compared using a one-way ANOVA, post hoc Tukey-Kramer test, and Student's $t$ test for independent samples ( $\mathrm{R}$ version
3.5.0; JMP 11.0.0, SAS Institute Inc., NC USA). Head/thorax mass was sampled for 10 bees per honeybee species and 25 differently sized bumblebee individuals, and the brain volume was measured for 8-9 bees per honeybee species and for 24 bumblebee workers. Neuron cell body volume and number were estimated for 5 honeybees per species and for 21 bumblebees (because the latter varied much more considerably in brain and body size).

\section{RESULTS}

\subsection{Brain-body size correlation}

Head/thorax mass did not differ much within any of the four honeybee species sampled, and no significant difference was found between honeybee species regarding the relative head/thorax mass variation (one-way ANOVA: $F=1.5$; $p=$ 0.26 ; variation between the smallest and the largest bee: A. florea $17.5 \%$; A. cerana $16 \%$; A. mellifera 19\%; A. dorsata 10\%; Figures 1a and $2 \mathrm{a}-\mathrm{d}$ ) whereas individuals of our B. impatiens sample differed by $676 \%$ (Figures 1a and 2e) and this variation was significantly different (Mares et al. 2005) compared with that of the honeybee species (Student's $t$ test; $p<10^{-15}$ ). Likewise, brain volumes did not differ much within each of the four honeybee species and only in A. florea showed a modest correlation with head/thorax mass $\left(R^{2}=0.79\right.$; Figure 2a) while brain volume differed strongly $(282 \%)$ among bumblebees and was highly correlated with head/thorax mass $\left(R^{2}=0.93\right.$; Figure $\left.2 \mathrm{e}\right)$.

Similar to the general allometric brain-body size relationship found in vertebrates (Jerison 1973), smaller honeybee species had relatively larger brains compared with their head/thorax mass (one-way ANOVA $F=17.23 ; p<0.0001$; Student $t$ test, $p<0.0001 ; n=56$; compare A. florea and A. dorsata in Figure 2f). Because of the large range of body and brain sizes in bumblebees, data for $B$. impatiens show a large standard deviation in Figure 2f; however, the smallest bumblebee had a body-to-brain ratio similar to A. florea (0.04) and the 10 largest bumblebees had a smaller body-to-brain ratio than the largest $A$. dorsata $(<0.021)$. As expected, brain 

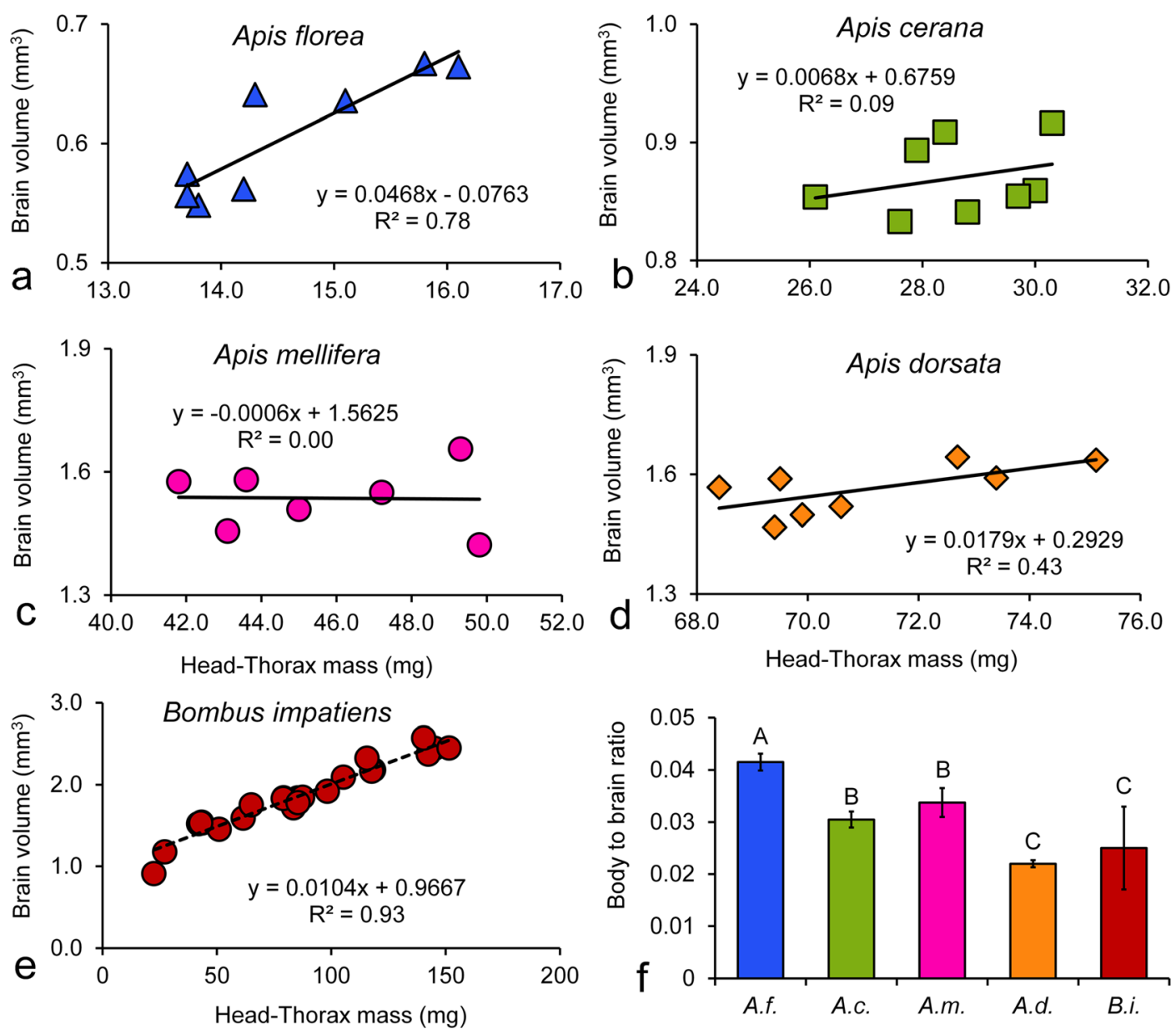

Figure 2. Correlation of head-thorax mass with brain volume plotted individually for each of the five bee species. a A. florea, $N=8$. b A. cerana, $N=8$. c A. mellifera,$N=7$. d A. dorsata,$N=8$. e B. impatiens, $N=25$. f Bodyto-brain ratio of honeybees and bumblebees; $N=56 ;$ A.f. Apis florea; A.c. A. cerana; A.m. A. mellifera; A.d. A. dorsata; B.i. Bombus impatiens. Letters indicate statistically significant difference (Student $t$ test, $p=0.0001$ ).

size systematically increased with body size when combining the data from all individual bees of the five bee species (ANCOVA: $F=430.02, \mathrm{df}=1$, $50, p<0.001)$.

While brain volume and head/thorax mass did not correlate within individual honeybee species (Figure $2 \mathrm{a}-\mathrm{d}$ ), we found a pronounced correlation when comparing across honeybee species (red line in Figure $3 \mathrm{a} ; p<0.0001$ ). While the four species did not overlap in head/thorax mass and the three Asian honeybee species also showed distinct, non-overlapping brain volumes (pairwise comparison; $p<10^{-7}$ to $10^{-10}$ ), A. mellifera on average had a brain size comparable to that of the larger $A$. dorsata (Figure 3a; mean brain volume A. mellifera $1.54 \pm 0.07 \mathrm{~mm}^{3} ;$ A. dorsata $1.56 \pm$ $0.06 \mathrm{~mm}^{3} ; p=0.85$, respectively). Hence, A. mellifera are characterized by having larger brains than expected for their body size when compared with the Asian honeybee species. Another interpretation of the data set in Figure 1a would be that the brain size of A. mellifera follows the trend of the two smaller bee species and that $A$. dorsata have comparatively smaller brains than the other bee species. However, calculating the linear regressions for just three honeybee species, omitting either A. mellifera or A. dorsata, respectively (broken lines in Figure 3a), results in 

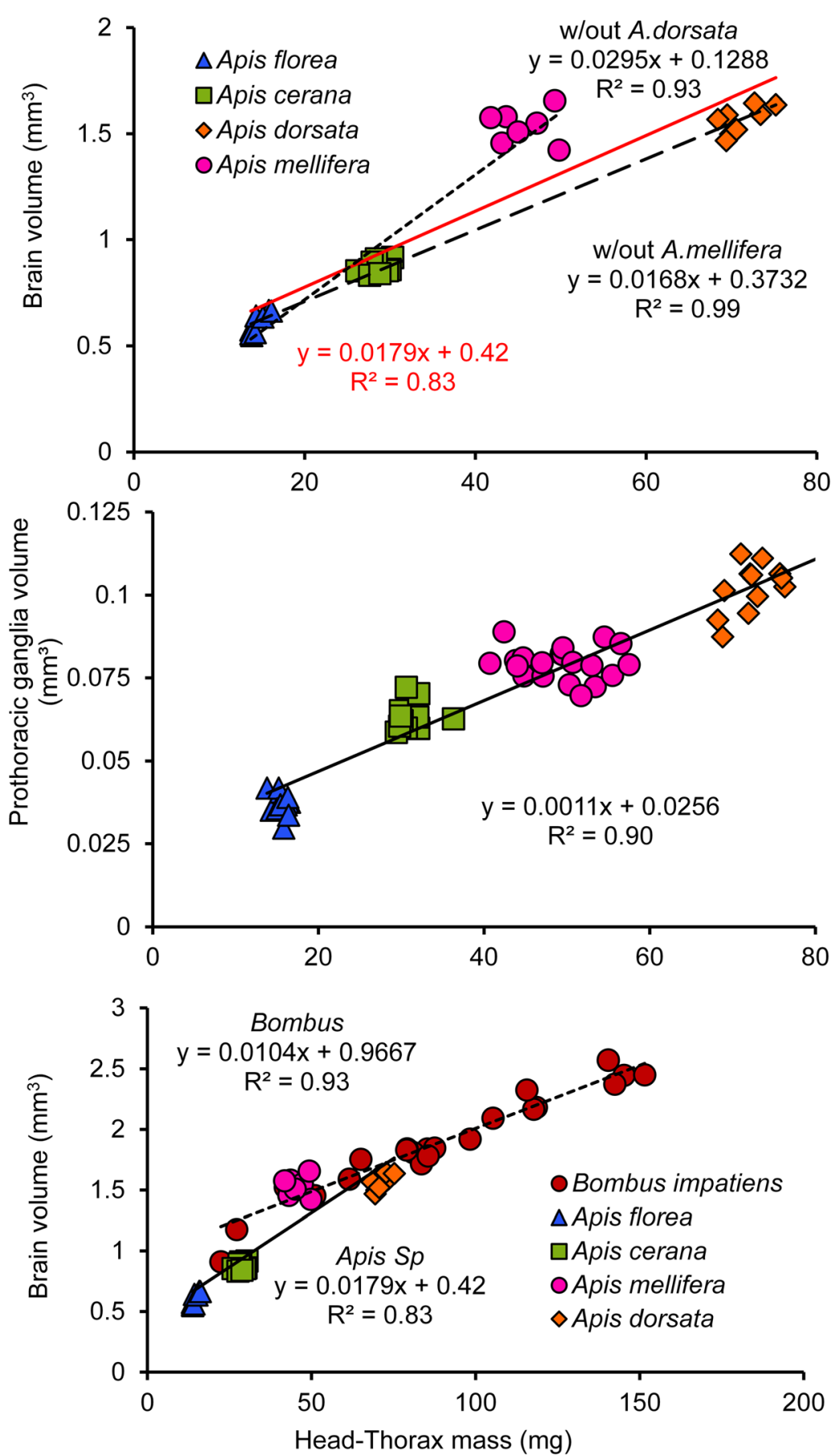

Figure 3. Correlation of head-thorax mass with a brain volume $(N=30)$ and $\mathbf{b}$ with thoracic ganglion volume, respectively $(N=55)$ of the four honeybee species combined. c Correlation of head-thorax mass with brain volume of all five bee species combined $(N=56)$. In (a), the red line indicates the linear regression for the correlation of all four honeybee species while the broken lines indicate linear regressions for just three species, omitting either A. mellifera or A. dorsata, respectively. In (c), solid line indicates linear regression for honeybees (same data as in (a)) and broken line indicates linear regression for bumblebees. See text for details. 
a slightly higher $R^{2}$ value (better fit) when omitting A. mellifera $\left(y=0.017 x+0.37 ; R^{2}=0.99\right)$ than when omitting $A$. dorsata $(y=0.03 x+0.13$; $R^{2}=0.93$ ), suggesting that $A$. mellifera is indeed the outlier and has relatively larger brains compared with the other honeybee species.

To test if A. mellifera's "outlier" status was restricted to the brain or if their nervous system is, for whatever reason, overall larger than expected, we compared the prothoracic ganglia of the four honeybee species. Figure $3 \mathrm{~b}$ shows that the relationship between prothoracic ganglion volume and head/thorax mass is strong and significant for all four honeybee species [Figure $3 b ; y=0.001 x+$ 0.025; $R^{2}=0.90$ ANCOVA: $F=1249.82, \mathrm{df}=1$, $51, p<0.0001$ (body size) $F=11.04, \mathrm{df}=1,51$, $p<0.0001$ (species)], and no significant interaction effect was found between head/thorax mass and species (ANCOVA: $F=1.0, \mathrm{df}=1,48, p=$ $0.39)$. The ganglion volumes do not overlap among species; specifically, the volume data for A. mellifera $\left(0.079 \pm 0.005 \mathrm{~mm}^{3}\right)$ and A. dorsata $\left(0.10 \pm 0.007 \mathrm{~mm}^{3}\right)$ are distinct and different (pairwise comparison, $p<0.0001$ ), unlike the respective species' brain volumes.

Including bumblebees and combining the data for the five individual bee species (Figure 2a-e) into a common graph (Figure 3) show that brainbody size correlations in bumblebees follow the same trend shown for the honeybee species (compare Figure 3a, c). Correlations between the bees' head widths and brain volumes were similar to correlations between head/thorax mass and brain volumes but with slightly lower $R^{2}$ values. We also calculated the brain-head-thorax mass data shown in Figure 3 on a double-logarithmic scale. For the four honeybee species, the slopes of the resulting regressions are as follows: $\ln _{\text {brain mass }}=$ $0.65 \times \ln _{\text {body mass }} ; R^{2}=0.92$ and $\ln _{\text {thoracic ganglion }}$ mass $=0.62 \times \ln _{\text {body mass }} ; R^{2}=0.93$; combining all five bee species, the slope is $\ln _{\text {brain mass }}=0.61 \times$ $\ln _{\text {body mass }} ; R^{2}=0.93$.

\subsection{Relative size of brain components}

We found significant differences between the five bee species regarding the relative size of all brain components examined (Figure 4), but for the central body, the antennal lobes, and the lobula, 
these differences were small (although statistically significant) and we will only point out the larger differences in Figure 4, which may potentially be of more functional relevance.

The most prominent difference was the relative size of the lamina of $A$. dorsata, which was much larger than in the other species (Figure 4; one-way ANOVA $F=150.9 ; p<0.0001$; Tukey-Kramer test $p<0.0001, n=56$ ). However, the lobula and medulla were relatively smaller in A. dorsata compared with the other species [Figure 4; (lobula oneway ANOVA $F=14.88 ; p<0.0001$; TukeyKramer test $p<0.0001, n=56$ ); medulla oneway ANOVA $F=19.68 ; p<0.0001$; TukeyKramer test $p<0.0001, n=56$, respectively)]. Compared with the larger honeybee species, the small $A$. florea invested less in antennal lobe and more in optic lobe neuropils (Figure 4).

The relative size of the MB calyx and the lobes and peduncle was similar in the four honeybee species, but both structures were considerably larger in the bumblebees (Figure 4; one-way ANOVA $F=95.12$ and 21.23 , respectively; $p<0.0001$, $n=56$ ). While A. mellifera had larger overall brains than expected for their body size (Figure 3a), their brain composition did not show any notable differences compared with the three Asian bee species (Figure 4). We also calculated relative neuropil volumes with respect to the overall neuropil volume (as opposed to overall brain volume), and those data confirmed the findings for neuropil volumes relative to total brain volume (suppl. Fig. 2).

\subsection{Cell body number and volume estimates}

A linear relationship existed between head/ thorax mass and the volume (Figure 5a; $y=$ $\left.2.96 x+313.91 ; R^{2}=0.59\right)$ as well as the number of antennal lobe cell bodies across all the five species (Figure 5b; $y=90.30 x+14,229 ; R^{2}=$ $0.67)$. The correlation between head/thorax mass and estimated antennal lobe cell body volume was stronger for the four Apis species (Figure 5a; $y=$ $\left.4.93 x+197.27 ; R^{2}=0.72\right)$. No significant

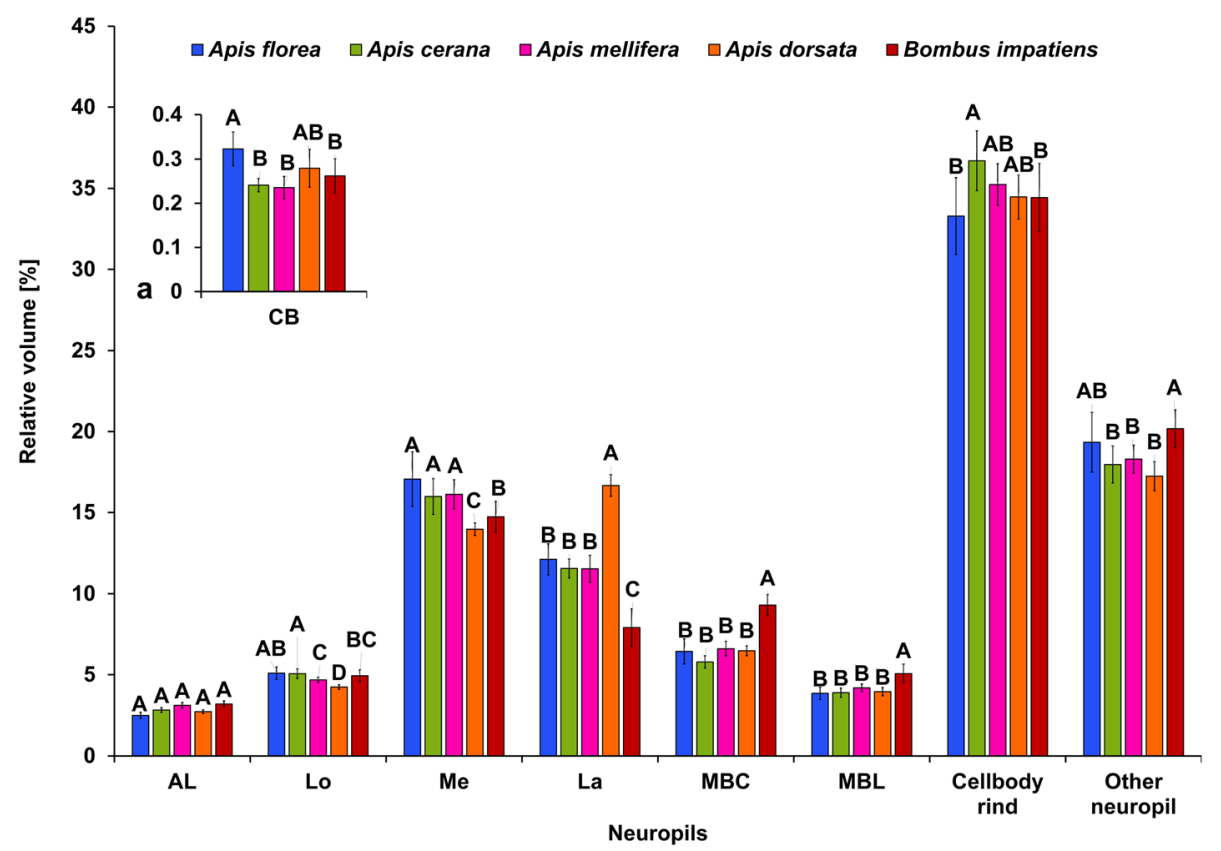

Figure 4. Relative volume of major brain components of honeybees and bumblebees $(N=56)$; letters indicate statistically significant differences (post hoc Tukey-Kramer test, $p=0.0001$ ); $A L$ antennal lobe; $L a$ lamina; $L o$ lobula; $M B C \mathrm{MB}$ calyx; $M B L \mathrm{MB}$ lobes and peduncle; $M e$ medulla. 

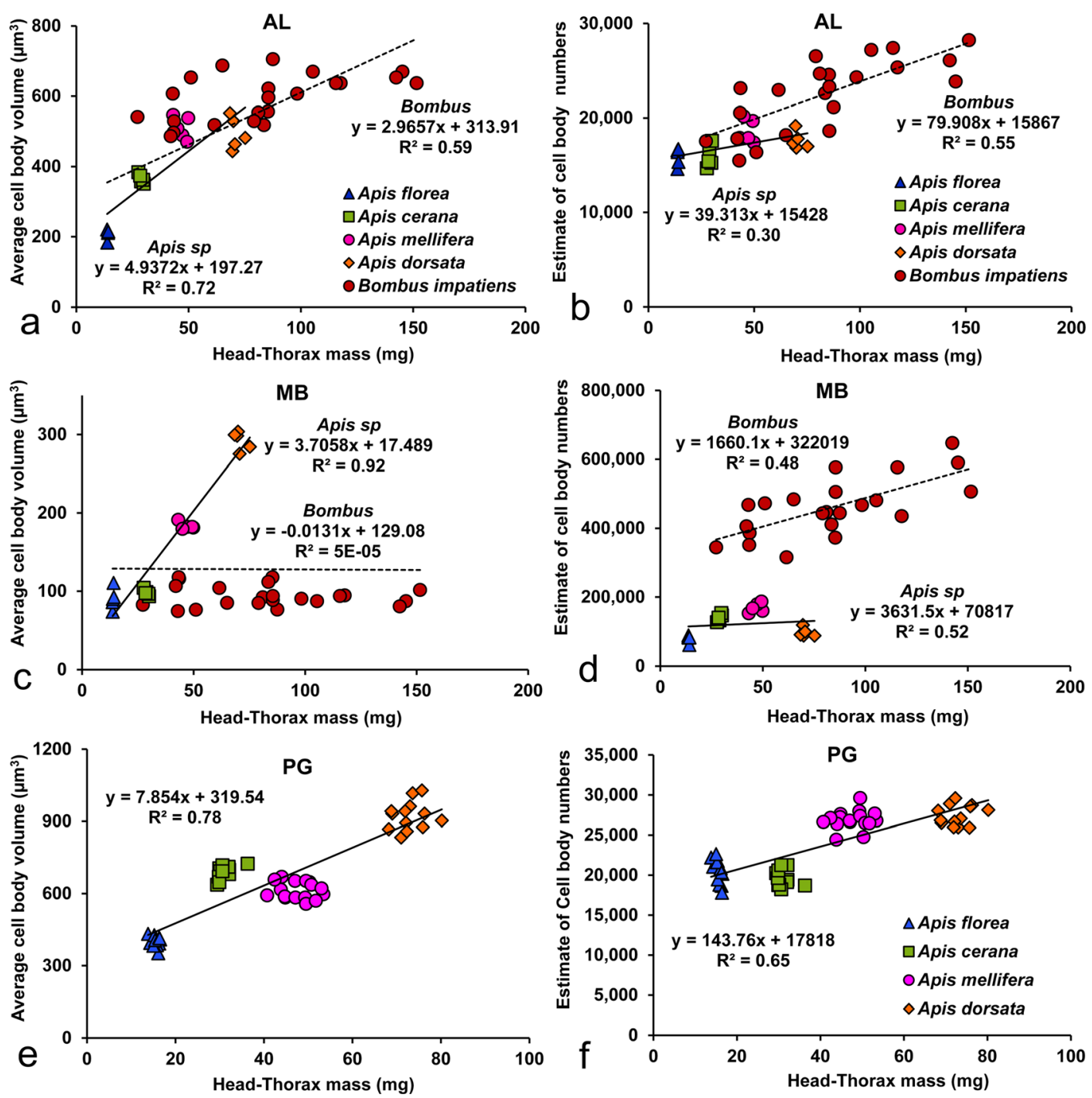

Figure 5. Comparison of estimated average cell body volume $(\mathbf{a}, \mathbf{c}, \mathbf{e})$ and average neuron cell body numbers $(\mathbf{b}$, d, f) of neurons associated with one antennal lobe $(N=40)(\mathbf{a}, \mathbf{b})$; neurons associated with lateral MB calyx $(N=$ 40) (c, d ); and neurons associated with the prothoracic ganglia $(N=48)(\mathbf{e}, \mathbf{f})$. (a-d $)$ include all five bee species studied; $(\mathbf{e}, \mathbf{f})$ only include the honeybee species. Letters indicate statistically significant differences (Tukey-Kramer test; $p=0.0001$ ); solid lines indicate linear regressions for bumblebees; broken lines indicate linear regressions for honeybees.

interaction between head/thorax mass and species was found for either estimated antennal lobe cell body volume or cell body number (ANCOVA: $F=0.48,0.28 ; \mathrm{df}=4,32, p=0.74,0.88$, respectively). The two species that had larger antennal lobes (Figure 4) also had significantly more (B. impatiens; one-way ANOVA $F=9.47$, $p<0.0001$; Tukey-Kramer test $p<0.0006, n=$ 40 ) or slightly (but not statistically significantly; A. mellifera) more estimated antennal lobe cell bodies compared with the Asian honeybee species (Figure 5b).

A strong correlation existed between head/ thorax mass and MB cell body volume of the four 
honeybee species $\left[y=3.70 x+17.48, R^{2}=0.92\right.$; Figure 5c; Table I]. In contrast, such a correlation did not exist for bumblebees $(y=-0.013 x+$ 129.08, $R^{2}=4.9 \mathrm{E}-05$; ANCOVA: $F=515, \mathrm{df}=$ $1,32, p<0.0001$; Figure $5 \mathrm{c}$ ), suggesting that estimated MB cell body volume increases with the body size across the four Apis species, but remains the same in bumblebees (B. impatiens). A strong correlation was found between MB neuron number and head/thorax mass (Figure $5 \mathrm{~d}, y=$ $\left.3631.5 x+70,817, R^{2}=0.52\right)$. Interestingly, the MB Kenyon cell volume increased profoundly with body size among Apis species $\left(R^{2}=0.92\right.$; Figure $5 \mathrm{c}$ ), whereas the Kenyon cell number did not increase with body size $\left[R^{2}=0.02\right.$; Figure $5 \mathrm{~d}$; Table I]. This contrasts with bumblebees, which had substantially and significantly more Kenyon cells compared with the four honeybee species [Figure 5d; $y=3631.5 x+70,817, R^{2}=0.52$; one-way ANOVA $F=75.71$; Tukey-Kramer test $p<0.0001 ; n=40$; Table I]. Despite their considerably larger body and MB size, bumblebee Kenyon cell bodies were estimated to be as small as those of the small honeybees $A$. cerana and $A$. florea (Figure $5 \mathrm{c}$ ). This was true for both, small and large Kenyon cell types (Table I).

Head/thorax mass and both prothoracic neuron volume and number were strongly correlated across the honeybees (Figure 5e; $y=7.85 x+$ $319.54, y=143.76 x+17,818 ; R^{2}=0.78,0.65$, respectively). The two larger honeybee species A. mellifera and A. dorsata had significantly more prothoracic neurons compared with the smaller A. florea and A. cerana (Figure 5e; oneway ANOVA $F=134.34$; Tukey-Kramer test $p<0.0001 ; n=52$ ). Prothoracic cell bodies were distinct in size for each honeybee species (oneway ANOVA $F=380.17, p<0.0001$; TukeyKramer test $p<0.0001 ; n=52$ ) with cell bodies of A. florea being the smallest and those of A. dorsata being the largest (Table I). However, the larger A. mellifera had smaller prothoracic cell bodies than the smaller A. cerana.

\section{DISCUSSION}

Our data show a general allometric correlation between body, brain, and prothoracic ganglia size and the volume and number of neurons. The brain composition is generally similar across the five bee species examined. Notable exceptions to these trends include the brains of Apis mellifera being relatively larger than expected and the lamina of A. dorsata and the MB of bumblebees being larger than expected. Bumblebees also feature smaller and substantially more MB neurons compared with the other bee species.

While an exponential allometric relationship exists between body and brain mass in vertebrates (Jerison 1973) and probably in animals in general, we found only a weak correlation between head/ thorax mass and brain volume within some of the honeybee species investigated (Figure 2). This is probably due to the relatively small differences in body size within those species. However, the brain-body size correlation is much more obvious in bumblebees (Figure 2e) and when comparing across bee species (Figure 3). A micro-CT study by Smith et al. (2016) describes the volumetric composition of a different bumblebee species, $B$. terrestris . Overall, this study (Smith et al. 2016) shows smaller brain components than our study or others by Mares et al. (2005) or Jones et al. (2013) showed for B. impatiens. Unfortunately, no total brain volumes were given in Smith et al. (2016), and the coefficient of determination $\left(R^{2}\right)$ is smaller in that study compared to other studies. We would have expected $B$. terrestris to have similar or slightly larger brains and brain components than $B$. impatiens, based on $B$. terrestris' slightly larger body size (Smith et al. 2016).

Interestingly, for the smallest insects (some tiny parasitoid wasps and some tiny ants), the brains are smaller than expected from the general allometric relationships found in larger animals [van der Woude et al. (2013); Seid et al. (2011)]. It appears that for the smallest insects, the metabolic expenses for maintaining an "appropriately" sized brain (which would be disproportionally large compared to larger animals) are too high for their small body size (van der Woude et al. 2013). However, even the smallest bees in our sample (A. florea) are still much larger than those tiny insects and follow the general allometric brainbody size relationship.

While only few other insect brain mass data have been published, based on just three insect 
species, Chittka and Niven (2009) suggested that insect brain/body size trends match those established for vertebrates. Transforming our data set (Figure 3c) logarithmically results in the following slope: $\ln _{\text {brain mass }}=0.61 \times \ln _{\text {body mass }} ; R^{2}=$ 0.93 , nesting the scaling exponent of our five bee species between mammals (0.78) and birds (0.58) as compiled by Wehner et al. (2007). Those authors (Wehner et al. 2007) found a correlation similar to ours comparing 10 related ant species $\left(\ln _{\text {brain mass }}=0.57 \times \ln _{\text {body mass }}-6.1\right)$. Seid et al. (2011) found different allometric coefficients for small ant species $(b=0.7961)$ compared to larger ant species $(b=0.669)$. Together, these different data sets suggest that similar scaling rules might apply to vertebrates and insects.

Our data of relative neuropil sizes are very similar to those published in other studies. Data for A. mellifera match very well those of the general honeybee brain atlas by Brandt et al. (2005). For example, the antennal lobe is $2.9 \%$ of the total neuropil volume in those authors' data and it is $3.1 \%$ in our data (likewise: the medulla is $15 \%$ vs. $16.1 \%$, the lobula is $4.3 \%$ vs. $4.7 \%$, the mushroom body lateral calyx is $3.5 \%$ vs. $3.4 \%$, and the medial calyx is $3.22 \%$ vs. $3.17 \%$ ). Our absolute volumes are higher than those in the Brandt et al. (2005) study, but it is generally not possible to compare absolute brain values across different studies because of different fixation techniques and optical approaches. For instance, the Brandt et al. (2005) study used methylsalicylate for brain clearing, a compound known to lead to strong tissue shrinkage (Bucher et al. 2000).

\subsection{European honeybees have large brains}

Following this general trend, we would have expected $A$. mellifera to have smaller brains than the larger $A$. dorsata (Figure 1a). The brain vs. head/thorax mass correlations fall on tight regression lines for A. florea, A. cerana, and A. dorsata (Figure $3 \mathrm{a}$ ), whereas $A$. mellifera data are situated above this line, indicating that $A$. mellifera differ from the other three closely related species in having relatively larger brains than expected by allometric scaling. However, little is known about how brain size relates to behavior in bees (or in most animals for that matter) and there are very few studies comparing the behavior of A. mellifera and Asian honeybee species (Dyer and Seeley 1991; Hepburn and Radloff 2011). The larger brain size of $A$. mellifera might potentially suggest more specialized behavioral or cognitive capacities, but there is currently no behavioral evidence to support such claims. A. mellifera queens are considerably larger than workers while this difference is less pronounced in A. dorsata. It is possible that queen brains of those two species might fit the overall regression line (Figure 3a) and that in A. mellifera the workers have a reduced body size (but not brain size) while the opposite might be the case for the robust workers of $A$. dorsata. While this might explain the differences from the expected brain sizes, in the absence of queen brain sizes, no evidence exists to support this speculation.

\subsection{Giant honeybees seem adapted for crepuscular vision}

Perhaps, the most striking differences in brain composition were found in the relative size of the lamina, which was considerably larger in A. dorsata compared with the other bees whereas the upstream visual centers (the medulla and lobula) were relatively smaller in this species (Figure 4). The lamina is the first optic neuropil and processes local vs. overall light intensity and contrast and reduces noise from the photoreceptor output, among other functions (Ribi 1975; Strausfeld 2012). Apis dorsata are diurnal as well as crepuscular foragers with eyes adapted for increased light sensitivity (relatively large eyes and more ommatidia than the other honeybee species (Somanathan et al. 2009)) and were therefore expected to feature large laminae. However, they had the relatively smallest medullae of all the bees studied, which was less expected as the medulla processes all the input from the lamina. Why would a bee with large eyes and a large lamina have a smaller medulla?

Hints may come from studies comparing diurnal and nocturnal bees Megalopta genalis (Greiner et al. 2004) and Xylocopa tranquebarica (Somanathan et al. 2008; Somanathan et al. 2009) or Lepidoptera (Stöckl et al. 2016a) which, in 
addition to retinal and optical adaptations, feature neuronal specializations supporting their ability of nocturnal vision. By analogy, the increased light sensitivity of $A$. dorsata might be achieved by tangential collaterals pooling the input from several ommatidia at the level of lamina monopolar cells (Greiner et al. 2004; Strausfeld 2012). Our data support this hypothesis as additional or enlarged lamina tangential neurons would extend the size of the lamina, and a reduction in the number of output neurons from the lamina would result in fewer channels (columns) in the medulla and lobula, thus potentially explaining the reduced size of these neuropils. Likewise, Stöckl et al. (2016b) report slight (not statistically significant) differences in lamina size between a diurnal and a nocturnal hawkmoth, but they found a significantly larger medulla, lobula, and lobula plate in the diurnal moth. The effect is similar to what we report here for A. dorsata : in the nocturnal insect, input from a relatively large lamina is processed by substantially smaller downstream visual neuropils in the nocturnal insect. Further neuroanatomical studies would be required to confirm this idea.

\subsection{Bumblebees have large mushroom bodies}

The MB were substantially and significantly larger in bumblebees compared with the honeybee species examined (Figure 2). This has also been found in an earlier study comparing A. mellifera and B. impatiens (Mares et al. 2005). MB are involved in behavioral modulation based on experience and learning and memory [reviewed in (Strausfeld 2012)]. Farris and Schulmeister (2011) suggest that MB have evolved in Hymenoptera based on the cognitive demands of host-finding behavior in parasitoids, particularly the capacity for associative and spatial learning, rather than in the context of sociality, but this finding does not explain the differences of MB size between honeybees and bumblebees. Bumblebees can learn more specialized flower handling abilities than honeybees [e.g., nectar robbing (Goulson et al. 2013)], and they can also learn new tasks by watching conspecifics [referred to as social learning (Alem et al. 2016; Worden et al. 2005)], advanced behaviors that may involve the MB.
The bumblebees' larger MB may potentially support more advanced sensory associations or discrimination (Galizia 2014) and cognitive flexibility (Loukola et al. 2017), but comparative behavioral evidence to support this notion is scarce.

Irrespective of the ultimate consequences of having enlarged MB (Mares et al. 2005), it is interesting that bumblebees feature considerably smaller MB neuron cell bodies, but not antennal lobe neuron cell bodies (Figure 5b) than expected for their body size (Figure 3c). The combination of enlarged $\mathrm{MB}$ and decreased $\mathrm{MB}$ neuron volume explains why bumblebees feature those particularly large numbers of Kenyon cells shown in Figure 5c [about 2.6 times as many as in A. mellifera, the honeybee species with the most Kenyon cells (Table I). It is the number of neurons that contributes to the computational capacities of brains more than the overall volume of a brain or neuropil (Herculano-Houzel 2009), and one might therefore potentially expect to find increases in cognitive abilities in bumblebees compared with honeybees.

\subsection{Prothoracic ganglia scale with body size}

While the brain represents an animal's advanced cognitive capacities, the ventral nerve cord integrates basic sensory and motor functions at the local level and therefore seems potentially suited to test basic size correlations between the body and the nervous system in insects, unencumbered by the factors that govern brain elaboration and cognitive abilities. The prothoracic ganglion seems particularly suited for this kind of comparisons as it mainly controls the forelegs and is therefore not involved in potential interspecific differences in flight capabilities. Our data confirm a highly significant correlation between head/ thorax mass and total prothoracic ganglion size (Figure $3 \mathrm{~b}$ ) and therefore represent a well-suited measure to serve as a standard to normalize brain size in comparative analyses. Specifically, the tight correlation between head/thorax mass and prothoracic ganglion volume in honeybees (Figure $3 \mathrm{~b}$ ) suggests that the relatively larger brains of $A$. mellifera (Figure $3 \mathrm{a}$ ) represent functional differences in this species, rather than an overall trend for enlarged neuropil, neuron volume, or neuron number (Table I). 


\section{CONCLUSION}

As is the case in vertebrates, we found an overall allometric relationship between body size and brain and brain component size, ganglion volume and neuron volume, and number across the five bee species examined. Individual differences in these measures point towards species-specific sensory or cognitive disparities. The relatively larger brain of A. mellifera, the particularly large $\mathrm{MB}$ and high number of associated neurons of $B$. impatiens, and the potential neuronal adaptations of $A$. dorsata for dim-light vision all imply behavioral capabilities that should be further examined in comparative behavioral experiments.

\section{ACKNOWLEDGMENTS}

We thank Yeshwanth, H. M at the University of Agricultural Sciences, Bangalore, India, and Lakshy Katariya at the Center for Ecological Sciences, Indian Institute of Science, Bangalore, India, for providing Asian bees; Gloria De-Grandi Hoffman at the USDA ARS Carl Hayden Bee Research Center, Tucson, Arizona, for granting access to collect European honeybees; and Illiana Manjon, Francisco Enverga, and Roopa Gowda for help with brain sectioning and drawing. We also thank Nicholas Strausfeld for helpful suggestions and advice during the development of the project and Rebekah Keating-Godfrey for helpful comments on the manuscript.

\section{AUTHORS' CONTRIBUTION}

VG and WG conceived the study, VG collected the data and performed the analyses, and VG and WG interpreted the analysis and wrote the manuscript.

\section{FUNDING INFORMATION}

This work was supported by student research grants from the Society for Integrative and Comparative Biology and the Graduate and Professional Student Council and the Center for Insect Science at the University of Arizona to VG and by the National Science Foundation grant IOS1354191 to WG.

\section{COMPLIANCE WITH ETHICAL STANDARDS}

Competing interests The authors declare that they have no competing interests.

Organisation et taille du cerveau chez des abeilles dont la taille corporelle diffère

mise à l'échelle allométrique / morphométrie / corps pédonculé / abeilles mellifères / bourdons

Organisation und Grösse des Gehirns bei sozialen Bienen von unterschiedlicher Körpergrösse

Allometrische Skalierung / Morphometrie / Pilzkörper / Honigbienen / Hummeln

\section{REFERENCES}

Alem, S., Perry, CJ., Zhu X, Loukola, OJ., Ingraham, T., Søvik, E., Chittka, L. (2016) Associative mechanisms allow for social learning and cultural transmission of string pulling in an insect. PLoS Biol. 14:e1002564.

Brandt, R., Rohlfing, T., Rybak, J., Krofczik, S., Maye, A., Westerhoff, M., Hege, H-C., Menzel R. (2005) Three-dimensional average-shape atlas of the honeybee brain and its applications. J. Comp. Neurol. 492:1-19.

Bucher, D., Scholz, M., Stetter, M., Obermayer, K., and Pflüger, H. J. (2000). Correction methods for threedimensional reconstructions from confocal images: I. Tissue shrinking and axial scaling. J. Neurosci. Methods 100, 135-143.

Byrne, RW., Bates, LA. (2007) Brain Evolution: When Is a Group Not a Group? Curr. Biol. 17, R883-R884.

Chittka, L., Niven, J, (2009) Are Bigger Brains Better? Curr. Biol. 19, R995-R1008.

Dunbar, RIM., Shultz, S. (2007) Evolution in the social brain. Science. 80 (317), 1344-1347.

Dyer, FC., Seeley, TD. (1991) Nesting Behavior and the Evolution of Worker Tempo in Four Honeybee Species. Ecology. 72,156-170.

Fahrbach, SE. (2006) Structure of the MB of the insect brain. Annu. Rev. Entomol. 51, 209-232.

Farris, MS., Schulmeister, S. (2011) Parasitoidism, not sociality, is associated with the evolution of elaborate 
mushroom bodies in the brains of hymenopteran insects. Proc. R. Soc. B 2011 278, 940-951; doi: https://doi.org/10.1098/rspb.2010.2161

Galizia, CG. (2014) Olfactory coding in the insect brain: Data and conjectures. Eur. J. Neurosci. 39, 1784-1795.

Goulson, D., Peat, J., Stout, JC., Tucker, J., Darvill, B., Derwent, LC., Hughes, WOH. (2002) Can alloethism in workers of the bumblebee, Bombus terrestris, be explained in terms of foraging efficiency? Anim. Behav. 64, 123-130.

Goulson, D., Park, KJ., Tinsley, MC., Bussière, LF., Vallejo-Marin, M. (2013) Social learning drives handedness in nectar-robbing bumblebees. Behav. Ecol. Sociobiol. 67, 1141-1150.

Greiner, B., Ribi, WA., Warrant, EJ. (2004) Retinal and optical adaptations for nocturnal vision in the halictid bee Megalopta genalis . Cell Tissue Res. 316, 377-390.

Gundersen, H., Jensen, E. (1987) The efficency of system sampling in stereology and its prediction. J. Microsc. 147, 229-263.

Healy, SD., Rowe, C. (2007) A critique of comparative studies of brain size. Proc. R. Soc. B-Biological. Sci. 274, 453-464.

Hepburn, HR., Radloff, SE. (2011) Honeybees of Asia. Springer-Verlag Berlin Heidelberg.

Herculano-Houzel, S. (2009) The human brain in numbers: a linearly scaled-up primate brain. Front. Hum. Neurosci. 3, 31.

Herculano-Houzel, S., Manger, PR., Kaas, JH. (2014) Brain scaling in mammalian evolution as a consequence of concerted and mosaic changes in numbers of neurons and average neuronal cell size. Front. Neuroanat. 8, 77.

Jerison, H. J. (1973) Evolution of the Brain and Intelligence. Curr. Anthropol. 16, 403-426.

Jones, BM., Leonard, AS., Papaj, DR., Gronenberg, W. (2013) Plasticity of the worker bumblebee brain in relation to age and rearing environment. Brain. Behav. Evol. 4, 250-261.

Lefebvre, L., Reader, SM., Sol, D. (2004) Brains, innovations and evolution in birds and primates. Brain Behav. Evol. 63, 233-246.

Loukola, OJ., Perry, CJ., Coscos, L., Chittka, L. (2017) Bumblebees show cognitive flexibility by improving on an observed complex behavior. Science 80 (355), 833-836.

Mares, S., Ash, L., Gronenberg, W. (2005) Brain allometry in bumblebee and honeybee workers. Brain Behav. Evol. 66, 50-61.

Martin, R. (1981) Relative brain size and basal metabolic rate in terrestrial vertebrates. Nature. 293, 57-60.

Neder, R. (1959) Allometrisches Wachstum von Hirnteilen bei drei verschieden grossen Schabenarten. Zoo. Jb. Anat. 77, 411-464.
Oldroyd, BP., Wongsiri, S. (2006) Asian honeybees: biology, conservation, and human interaction. Harvard University Press., Cambridge.

Ribi, WA. (1975) The first optic ganglion of the bee. I. Correlation between visual cell types and their terminals in the lamina and medulla. Cell Tissue Res.. 165,103-111.

Riveros, AJ., Gronenberg, W. (2010) Brain allometry and neural plasticity in the bumblebee Bombus occidentalis . Brain Behav. Evol., 75:138-148; DOI: https://doi.org/10.1159/000306506/.

Seid, MA., Castillo, A., Wcislo, WT. (2011) The Allometry of Brain Miniaturization in Ants. Brain Behav. Evol., 77:5-13 DOI: https://doi.org/10.1159/000322530.

Smith DB, Bernhardt G, Raine NE, Abel RL, Sykes D, Ahmed F, Pedroso I, Gill R (2016) Exploring miniature insect brains using micro-CT scanning techniques. Sci. Rep. 6:21768; DOI: https://doi.org/10.1038 /srep21768.

Somanathan, H., Borges, RM., Warrant, EJ., Kelber, A. (2008) Visual ecology of Indian carpenter bees I: Light intensities and flight activity. J. Comp. Physiol. A. Neuroethol. Sensory. Neural. Behav. Physiol. 194, 97-107.

Somanathan, H., Warrant, EJ., Borges, RM., Wallén, R., Kelber, A. (2009) Resolution and sensitivity of the eyes of the Asian honeybees Apis flore a, Apis cerana and Apis dorsata. J. Exp. Biol. 212, 2448-2453.

Stöckl, AL., Ribi, WA., Warrant, EJ. (2016a) Adaptations for nocturnal and diurnal vision in the hawkmoth lamina. J. Comp. Neurol. 524,160-175; DOI https://doi. org/10.1002/cne.23832.

Stöckl, A., Heinze, S., Charalabidis, A., el Jundi, B., Warrant, E., Kelber, A. (2016b) Differential investment in visual and olfactory brain areas reflects behavioural choices in hawk moths. Sci. Rep. 6, 26041; doi: https://doi.org/10.1038/srep26041.

Strausfeld, NJ. (2012) Arthropod brains: Evolution, Functional Elegance, and Historical Significance. Harvard University Press, Cambridge.

Striedter, GF. (2005) Principles of Brain Evolution. Sunderland., Sinauer Associates.

Wehner, R., Fukushi, T., Isler, K. (2007) On being small: brain allometry in ants. Brain Behav. Evol. 69:220-228.

Worden, BD., Skemp, AK., Papaj, DR. (2005) Learning in two contexts: the effects of interference and body size in bumblebees. J. Exp. Biol. 208, 2045-2053.

van der Woude, E., Smid, HM., Chittka, L., Huigens, ME. (2013) Breaking Haller's rule: brain-body size isometry in a minute parasitic wasp. Brain Behav. Evol. 81:86-92; DOI: https://doi.org/10.1159/000345945.

Publisher's note Springer Nature remains neutral with regard to jurisdictional claims in published maps and institutional affiliations. 